\title{
Strain Distribution Analysis of Sputter-Formed Strained Si by Tip-Enhanced Raman Spectroscopy
}

\author{
Hiroaki Hanafusa, Nobumitsu Hirose ${ }^{1}$, Akifumi Kasamatsu', Takashi Mimura ${ }^{1}$, Toshiaki Matsui ${ }^{1}$, \\ Harold M. H. Chong'², Hiroshi Mizuta², and Yoshiyuki Suda*
}

\author{
Graduate School of Engineering, Tokyo University of Agriculture and Technology, 2-24-16 Naka-cho, Koganei, Tokyo 184-8588, Japan \\ ${ }^{1}$ National Institute of Information and Communications Technology, 4-2-1 Nukui-Kitamachi, Koganei, Tokyo 184-8795, Japan \\ ${ }^{2}$ School of Electronic and Computer Science, University of Southampton, Highfield, Southampton SO17 1BJ, U.K. \\ Received November 1, 2010; accepted December 18, 2010; published online January 13, 2011
}

Simultaneous nanometer-scale measurements of the strain and surface undulation distributions of strained $\mathrm{Si}$ (s-Si) layers on strain-relief quadruple- $\mathrm{Si}_{1-x} \mathrm{Ge}_{x}$-layer buffers, using a combined atomic force microscopy (AFM) and tip-enhanced Raman spectroscopy (TERS) system, clarify that an s-Si sample formed by our previously proposed sputter epitaxy method has a smoother and more uniformly strained surface than an $\mathrm{s}$-Si sample formed by gas-source molecular beam epitaxy. The TERS analyses suggest that the compositional fluctuation of the underlying $\mathrm{Si}_{1-x} \mathrm{Ge}_{x}$ buffer layer is largely related to the weak s-Si strain fluctuation of the sputtered sample. (C) 2011 The Japan Society of Applied Physics

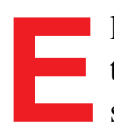
lectron and hole mobilities in $\mathrm{Si}$ are enhanced by the introduction of strain. Biaxial global-strain-type strained $\mathrm{Si}$ (s-Si) formed on a $\mathrm{Si}_{1-x} \mathrm{Ge}_{x}$ strain-relief relaxed buffer has been intensively applied to high-electronmobility transistors such as modulation-doped field-effect and metal-oxide-semiconductor transistors. ${ }^{1-5)}$

However, when we form global-strain-type s-Si by currently used gas-source methods such as gas-source molecular beam epitaxy (GS-MBE) and chemical vapor deposition (CVD), a crosshatch undulation pattern is generally formed on the surface with an undulation pitch of typically less than $1 \mu \mathrm{m} .{ }^{6)}$ This surface undulation causes a nonuniform strain distribution $^{7)}$ and variations in the physical properties and device performance on the surface. This is a serious issue to be solved for smaller and higher-density devices.

Recently, we have proposed a stepwise quadruple$\mathrm{Si}_{1-x} \mathrm{Ge}_{x}$-layer buffer (QL buffer) as a strain-relief relaxed buffer. ${ }^{8)}$ A smoother s-Si surface has been obtained on the QL buffer by our proposed sputter epitaxy method, which uses a combination of ultrahigh-vacuum-compatible magnetron sputtering and an $\mathrm{Ar} / \mathrm{H}_{2}$ mixture working gas, than by GS-MBE. ${ }^{9)}$ Therefore, this s-Si surface formed by our sputter epitaxy method is expected to have a more uniform strain distribution.

Raman spectroscopy is one of the useful techniques for evaluating the surface strain distribution; however, its conventional spatial resolution is limited to about $1 \times 1 \mu \mathrm{m}^{2}$ due to the diffraction limit and it is difficult to evaluate the s-Si surface strain distribution with an undulation pitch of $\lesssim 1 \mu \mathrm{m}$.

To obtain a higher spatial resolution, we have applied a near-field and plasmon oscillation coupling method ${ }^{10,11)}$ and introduced a combined atomic force microscopy (AFM) and tip-enhanced Raman spectroscopy (TERS) system for simultaneous measurements of surface topography and the enhanced Raman shift spectrum for an s-Si surface. So far, there has been one report, using a similar AFM-TERS system, on strain variation at the nanometer scale within a $1.4 \mu \mathrm{m}$ crosshatch segment on the s-Si surface on a $\mathrm{Si}_{1-x} \mathrm{Ge}_{x}$ graded buffer. ${ }^{11)}$

In this paper, using the AFM-TERS method, we first report the relationship between the surface roughness and

*E-mail address: sudayos@cc.tuat.ac.jp

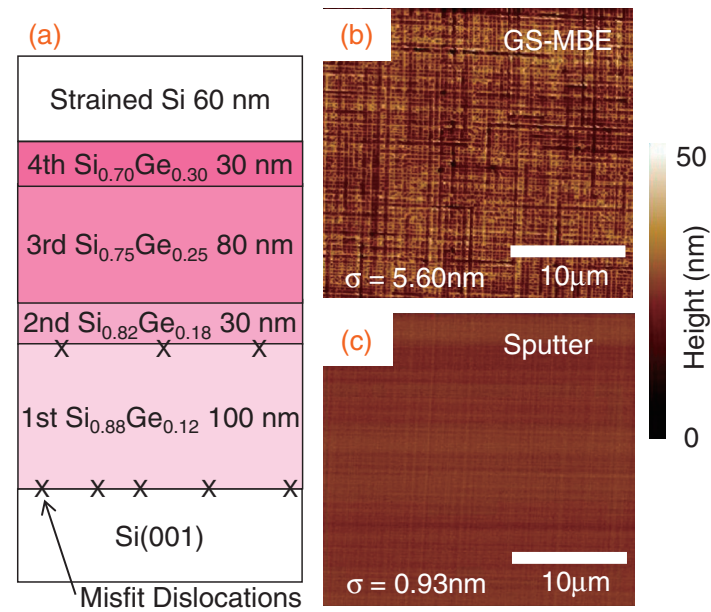

Fig. 1. (a) Structure of s-Si on our previously proposed QL strain-relief buffer, ${ }^{8)}$ and $30 \times 30 \mu \mathrm{m}^{2}$ AFM images of surfaces of strained Si formed by (b) GS-MBE and (c) our previously proposed sputter epitaxy method. ${ }^{9)}$

the strain distribution, on the nanometer scale, of strained Si on the stepwise QL buffer formed by our sputter epitaxy method. The results are then compared with those obtained with the GS-MBE method.

We show the sample structure in Fig. 1(a). Strain-relief QL buffers and $60 \mathrm{~nm}$ s-Si layers on the QL buffers were grown on $3-4 \Omega \mathrm{cm}$ p-type $\mathrm{Si}(001)$ by our sputter epitaxy method at a growth temperature, $T_{\mathrm{G}}$, of $500^{\circ} \mathrm{C}$ and by GS-MBE using $\mathrm{Si}_{2} \mathrm{H}_{6}$ and $\mathrm{GeH}_{4}$ as $\mathrm{Si}$ and $\mathrm{Ge}$ source gases, respectively, at $T_{\mathrm{G}}=600^{\circ} \mathrm{C}$. The $\mathrm{QL}$ buffers have a structure of $30 \mathrm{~nm} \mathrm{Si}_{0.7} \mathrm{Ge}_{0.3} / 80 \mathrm{~nm} \mathrm{Si}_{0.75} \mathrm{Ge}_{0.25} / 30 \mathrm{~nm}$ $\mathrm{Si}_{0.82} \mathrm{Ge}_{0.18} / 100 \mathrm{~nm} \mathrm{Si}_{0.88} \mathrm{Ge}_{0.12}$ on $\mathrm{Si}(001)$. The relaxation rate $R$ values of the third and fourth (top) buffer layers of the samples used in this work were 56 and $49 \%$ for the GSMBE sample, and 56 and $39 \%$ for the sputtered sample, respectively. The $R$ values of the fourth buffer layers vary between $\pm 5 \%$; however, all GS-MBE and sputtered samples exhibit undulated and flat surfaces under these experimental conditions, respectively.

The AFM-TERS measurements were carried out using an integrated system consisting of a Nanonics atomic force microscope and a Renishaw Raman spectrometer as illustrated in Fig. 2. In the AFM and TERS measurements, 


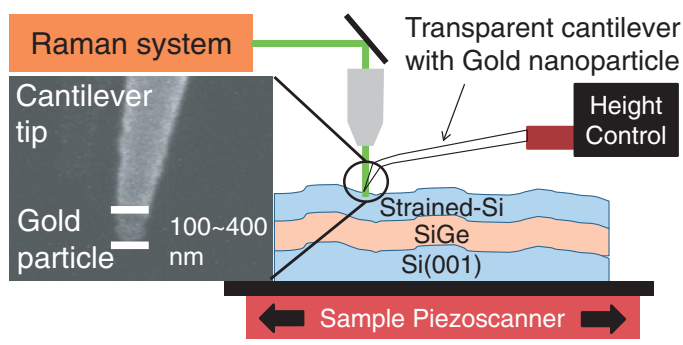

Fig. 2. Schematic of AFM-TERS system for simultaneous measurement of surface undulation profile and Raman shift spectrum for s-Si layer on $\mathrm{Si}_{1-x} \mathrm{Ge}_{x}$ multilayer buffer.

a transparent cantilever with a Au nanoparticle with a diameter of approximately $200 \mathrm{~nm}$ on its tip was used. A Nd-doped Y-Al-garnet laser (Nd:YAG) with a wavelength of $532 \mathrm{~nm}$ was used for excitation of surface plasmon oscillation around the $\mathrm{Au}$ nanoparticle. Thus, enhancement of the near field in the vicinity of the Au nanoparticle tip can be generated. ${ }^{10)}$

In Fig. 1, we show typical $30 \times 30 \mu \mathrm{m}^{2}$ AFM images of s-Si surfaces formed by (b) GS-MBE and (c) our sputter epitaxy method. A clear crosshatch undulation pattern is observed on the GS-MBE sample surface and the standard deviation $\sigma$ of the roughness distribution over the image was $5.6 \mathrm{~nm}$. It is also noted that a crosshatch undulation with $\sigma=4 \mathrm{~nm}$ has been reported with a $\mathrm{Si}_{1-x} \mathrm{Ge}_{x}$ graded buffer, graded up to $x=0.29$, formed by $\mathrm{CVD}$ at $T_{\mathrm{G}}=500^{\circ} \mathrm{C}$. ${ }^{12)}$ On the other hand, on the sputtered sample surface, the crosshatch undulation is very weak with $\sigma=0.93 \mathrm{~nm}$. The crosshatch pitches on both surfaces are approximately $400 \mathrm{~nm}$. However, the sputtered s-Si surface is smoother than the s-Si surface formed by GS-MBE. In the case of GSMBE using $\mathrm{Si}_{2} \mathrm{H}_{6}$ and $\mathrm{GeH}_{4}$, one-directional $60^{\circ}$ lattice plane slips are observed in the strain-relief buffer, ${ }^{9)}$ which results from the step-flow growth mode with hydride adsorbates ${ }^{13)}$ and causes a clear crosshatch undulation on the surface. However, with the sputter method, multidirectional $60^{\circ}$ lattice plane slips are observed, ${ }^{9}{ }^{9}$ which may result from more isotropic two-dimensional island nucleation with less movable nonhydrogenated $\mathrm{Si}$ and $\mathrm{Ge}$ adsorbates than the hydrides and forms a smoother surface.

We measured both the TERS spectra and the AFM morphologies along $1850 \mathrm{~nm}$ lines $\alpha-\alpha^{\prime}$ and $\beta-\beta^{\prime}$ on the GS-MBE and sputtered sample surfaces, respectively, as indicated in the AFM images shown in Fig. 3(a). The brighter areas indicate more convex regions on the surface. The lines pass through the crosshatch undulations for both the samples. The corresponding AFM profiles are shown in Fig. 3(b). The $\sigma$ values along $\alpha-\alpha^{\prime}$ and $\beta-\beta^{\prime}$ are 3.5 and $0.91 \mathrm{~nm}$, respectively, and show changes in undulation similar to those observed in the $30 \times 30 \mu \mathrm{m}^{2}$ areas indicated in Fig. 1.

The TERS Raman shift spectra measured at each point along $\alpha-\alpha^{\prime}$ and $\beta-\beta^{\prime}$ are shown in Fig. 4. Compared with the conventional Raman spectra, which are also shown at the bottom of the right and left figures, $\mathrm{Si}-\mathrm{Si}$ peaks from the s-Si and four $\mathrm{Si}_{1-x} \mathrm{Ge}_{x}$ QL buffer layers are clearly observed in the TERS spectra. The TERS Si-Si peak positions fluctuate with the measurement position. The $\mathrm{Si}-\mathrm{Si}$ peaks obtained
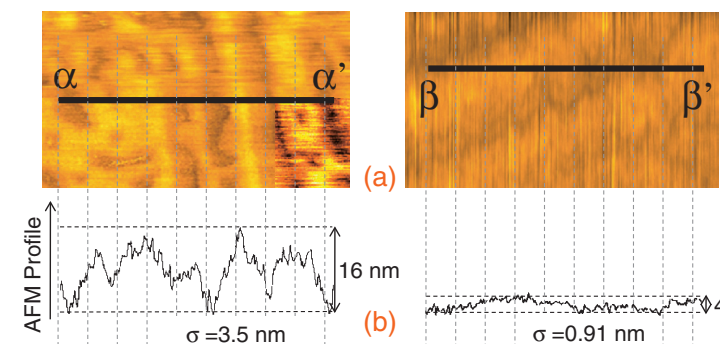

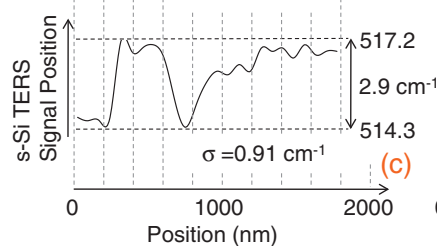

GS-MBE

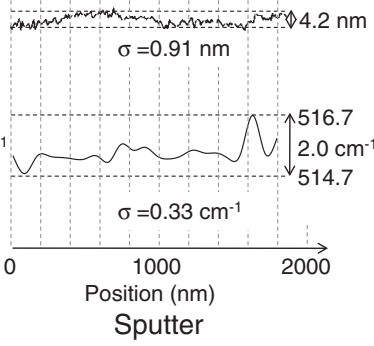

Fig. 3. (a) AFM images and $1850 \mathrm{~nm}$ measurement lines along which AFM profiles and TERS spectra are measured, (b) corresponding AFM profiles, and (c) corresponding $\mathrm{Si}-\mathrm{Si}$ TERS signal position profiles for s-Si samples formed by GS-MBE (left side) and sputter epitaxy (right side).

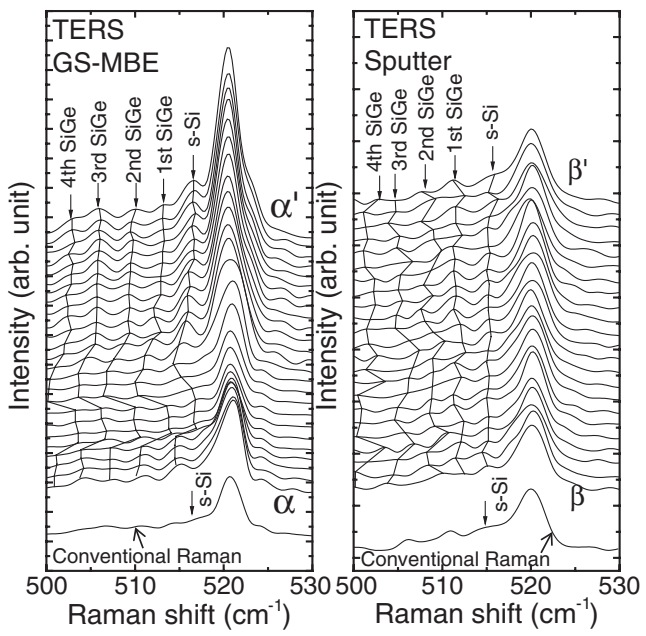

Fig. 4. Si-Si TERS spectra obtained at each position along lines $\alpha-\alpha^{\prime}$ and $\beta-\beta^{\prime}$ indicated in (a). Conventional Raman spectra are also shown at the bottom of the right and left figures.

from the s-Si layers are shown in Fig. 3(c). The large Raman-shift changes over a distance of $\sim 200 \mathrm{~nm}$ suggest that the TERS spatial resolution is at least on the order of $\sim 200 \mathrm{~nm}$. The $\sigma$ values of the s-Si peak positions along $\alpha-\alpha^{\prime}$ and $\beta-\beta^{\prime}$ are 0.91 and $0.33 \mathrm{~cm}^{-1}$, respectively. The Si-Si Raman shift in s-Si depends on the strain as described below. By comparing the AFM and Raman shift profiles in Figs. 3(b) and 3(c), the AFM peak height is found to be slightly related to the Raman shift, the surface flatness is strongly related to the strain uniformity of the s-Si layer, and the s-Si layer formed by our sputter epitaxy method has a more uniform strain distribution as well as a smoother surface than that formed by GS-MBE.

The Si-Si Raman shift in $\mathrm{Si}_{1-x} \mathrm{Ge}_{x}$ has been often analyzed to be related to the Ge composition $x$ and the lattice strain. ${ }^{14)}$ In this case, the $\mathrm{Si}-\mathrm{Si}$ Raman shift fluctuation in $\mathrm{Si}_{1-x} \mathrm{Ge}_{x}, \Delta \omega_{\mathrm{SiGe}}$, depends on the compositional fluctuation, $\Delta x$, and the strain fluctuation, $\Delta \varepsilon_{\mathrm{SiGe}}$ : $\Delta \omega_{\mathrm{SiGe}}=a \Delta x+b_{\mathrm{SiGe}} \Delta \varepsilon_{\mathrm{SiGe}}$, where $a$ and $b_{\mathrm{SiGe}}$ are the 


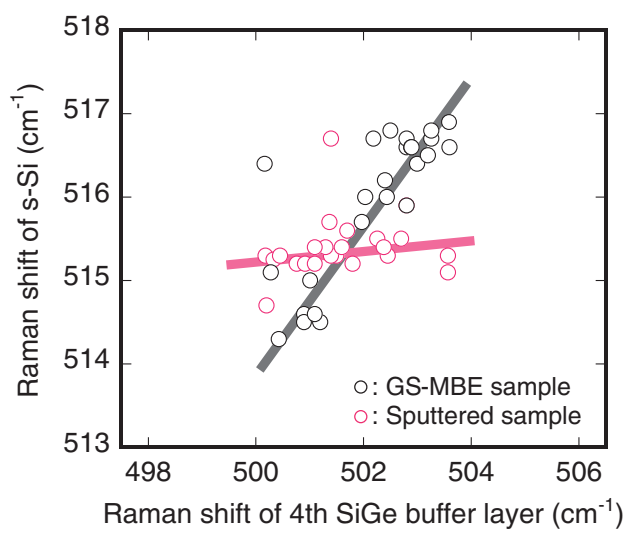

Fig. 5. Relationships between Si-Si Raman shifts of s-Si and the 4th $\mathrm{Si}_{0.7} \mathrm{Ge}_{0.3}$ QL buffer layer formed by GS-MBE and sputter epitaxy.

corresponding coefficients. ${ }^{15,16)}$ On the other hand, the $\mathrm{Si}-\mathrm{Si}$ Raman shift in Si depends on the lattice strain, and the $\mathrm{Si}-\mathrm{Si}$ Raman shift fluctuation in $\mathrm{s}-\mathrm{Si}, \Delta \omega_{\mathrm{Si}}$, reflects only the strain fluctuation, $\Delta \varepsilon_{\mathrm{Si}}: \Delta \omega_{\mathrm{Si}}=b_{\mathrm{Si}} \Delta \varepsilon_{\mathrm{Si}}$. We have analyzed the experimentally obtained Raman shift fluctuation data by assuming these relationships. The fluctuation $\Delta \omega_{\mathrm{Si}}$ in $\mathrm{s}-\mathrm{Si}$ is influenced by the fluctuation $\Delta \omega_{\mathrm{SiGe}}$ in the underlying $\mathrm{Si}_{1-x} \mathrm{Ge}_{x}$ layers and the $\Delta \omega_{\mathrm{Si}} / \Delta \omega_{\mathrm{SiGe}}$ ratio is given by $b_{\mathrm{Si}} \Delta \varepsilon_{\mathrm{Si}} /\left(a \Delta x+b_{\mathrm{SiGe}} \Delta \varepsilon_{\mathrm{SiGe}}\right) .{ }^{17)}$ If the fluctuation $\Delta \omega_{\mathrm{Si}}$ is related only to the underlying compositional fluctuation (i.e., $\Delta \varepsilon_{\mathrm{SiGe}}=0$ ), the $\Delta \omega_{\mathrm{Si}} / \Delta \omega_{\mathrm{SiGe}}$ ratios for the GS-MBE and sputtered samples are calculated to be 0.23 and 0.19 , respectively, using $\Delta \varepsilon_{\mathrm{Si}}=R \Delta x(c \mathrm{Ge}-c \mathrm{Si}) / c \mathrm{Si}$, where $c \mathrm{Si}$ and $c \mathrm{Ge}$ are the lattice constants of relaxed $\mathrm{Ge}$ and $\mathrm{Si}$, respectively, and $R$ values are 0.49 for the GS-MBE sample and 0.39 for the sputtered sample, as described in the experimental section above; $a=68 \mathrm{~cm}^{-115)}$ and $b_{\mathrm{Si}}=-784$ $\mathrm{cm}^{-1} \cdot{ }^{16)}$ If the fluctuation $\Delta \omega_{\mathrm{Si}}$ is related only to the underlying strain fluctuation (i.e., $\Delta x=0$ ), the $\Delta \omega_{\mathrm{Si}} / \Delta \omega_{\mathrm{SiGe}}$ ratio is calculated to be 0.83 using $\Delta \varepsilon_{\mathrm{Si}} / \Delta \varepsilon_{\mathrm{SiGe}}=c \mathrm{Si}_{0.7} \mathrm{Ge}_{0.3} /$ $c \mathrm{Si}$, where $c \mathrm{Si}_{0.7} \mathrm{Ge}_{0.3}$ is the lattice constant of relaxed $\mathrm{Si}_{0.7} \mathrm{Ge}_{0.3}$, and $b_{\mathrm{SiGe}}=-1043 \mathrm{~cm}^{-1}$ at $x=0.3{ }^{16)}$

From the results shown in Fig. 4, the relationships between the Si-Si Raman shifts of the s-Si layer and the 4th $\mathrm{Si}_{0.7} \mathrm{Ge}_{0.3}$ QL buffer layer are plotted in Fig. 5. The $\Delta \omega_{\mathrm{Si}} / \Delta \omega_{\mathrm{SiGe}}$ ratios correspond to the slopes of the lines indicated in the figure. The slopes are $0.84 \pm 0.10$ and $0.14 \pm 0.06$ for the GS-MBE and sputtered samples, respectively. The experimental results apparently show different slopes between the GS-MBE and sputtered samples. The $\Delta \omega_{\mathrm{Si}} / \Delta \omega_{\mathrm{SiGe}}$ ratio of the GS-MBE sample is close to the theoretical $\Delta \omega_{\mathrm{Si}} / \Delta \omega_{\mathrm{SiGe}}$ ratio for $\Delta x=0$, which suggests that the main origin of the s-Si strain fluctuation of the GS-MBE sample is attributed to the strain fluctuation of the underlying $\mathrm{Si}_{1-x} \mathrm{Ge}_{x}$ buffer layer. ${ }^{17)}$ The strain fluctuation is related to the crosshatch undulation. The $\Delta \omega_{\mathrm{Si}} / \Delta \omega_{\mathrm{SiGe}}$ ratio of the sputtered sample is $0.14 \pm 0.06$ and is smaller than the theoretical ratio of 0.83 for $\Delta x=0$. A factor which decreases the $\Delta \omega_{\mathrm{Si}} / \Delta \omega_{\mathrm{SiGe}}$ ratio considerably from that for $\Delta x=0$ is the underlying compositional fluctuation; thus, it is also suggested that the compositional fluctuation of the underlying $\mathrm{Si}_{1-x} \mathrm{Ge}_{x}$ buffer layer is largely related to the weak s-Si strain fluctuation of the sputtered sample. Even if the $R$ values change in the ranges of $0.49 \pm 0.05$ for our GS-MBE samples and $0.39 \pm 0.05$ for our sputtered samples, theoretical $\Delta \omega_{\mathrm{Si}} / \Delta \omega_{\mathrm{SiGe}}$ ratios for $\Delta \varepsilon_{\mathrm{SiGe}}=0$ vary within the ranges of $0.23 \pm 0.03$ and $0.19 \pm 0.03$, respectively. Thus, these $R$ changes do not affect the above suggestions. Since the crosshatch undulation is considered to be reduced by surface flattening by the multidirectional lattice plane slips in the case of the sputtered sample, ${ }^{9}$ the Raman analyses also suggest that multidirectional slips are initiated by the compositional fluctuation as well as by less movable adsorbates than hydrides. Further systematical study will clarify the precise factors which induce the slight discrepancy between the theoretical predictions and experimental results.

In conclusion, we first carried out nanometer-scale simultaneous measurements of the strain and surface undulation distributions for $\mathrm{s}-\mathrm{Si}$ layers on our proposed quadruple-Si $\mathrm{Si}_{1-x} \mathrm{Ge}_{x}$-layer buffers formed by GS-MBE and our sputter epitaxy method using a combined AFM-TERS system. The results show the strong relationship between the surface flatness and the strain distribution uniformity. The s-Si layer formed by our sputter epitaxy method has a smoother surface with a more uniform strain distribution than that formed by GS-MBE. The TERS analyses suggest that the compositional fluctuation of the underlying $\mathrm{Si}_{1-x} \mathrm{Ge}_{x}$ buffer layer is largely related to the weak $\mathrm{s}-\mathrm{Si}$ strain fluctuation of the sputtered sample, which may also be related to the multidirectional lattice plane slips which cause the surface flattening.

Acknowledgments The work reported here is partially supported by the International Training Program conducted by Tokyo University of Agriculture and Technology and the Japan Society for the Promotion of Science.

1) C. K. Maiti, L. K. Bera, and S. Chattopadhyay: Semicond. Sci. Technol. 13 (1998) 1225.

2) M. L. Lee, E. A. Fitzgerald, M. T. Bulsara, M. T. Currie, and A. Lochtefeld: J. Appl. Phys. 97 (2005) 011101.

3) K. Sawano, Y. Hoshi, A. Yamada, Y. Hiraoka, N. Usami, K. Arimoto, K. Nakagawa, and Y. Shiraki: Appl. Phys. Express 1 (2008) 121401.

4) N. Hirashita, Y. Moriyama, S. Nakaharai, T. Irisawa, N. Sugiyama, and S. Takagi: Appl. Phys. Express 1 (2008) 101401.

5) K. Sawano, Y. Kunishi, Y. Satoh, K. Toyama, K. Arimoto, T. Okamoto, N. Usami, K. Nakagawa, and Y. Shiraki: Appl. Phys. Express 1 (2008) 011401.

6) M. A. Lutz, R. M. Feenstra, F. K. LeGoues, P. M. Mooney, and J. O. Chu: Appl. Phys. Lett. 66 (1995) 724.

7) K. Sawano, N. Usami, K. Arimoto, S. Koh, K. Nakagawa, and Y. Shiraki: Mater. Sci. Semicond. Proc. 8 (2005) 177.

8) H. Maekawa, Y. Sano, C. Ueno, and Y. Suda: J. Cryst. Growth 301-302 (2007) 1017.

9) H. Hanafusa, A. Kasamatsu, N. Hirose, T. Mimura, T. Matsui, and Y. Suda: Jpn. J. Appl. Phys. 47 (2008) 3020.

10) L. Zhu, C. Georgi, M. Hecker, J. Rinderknecht, A. Mai, Y. Ritz, and E. Zschech: J. Appl. Phys. 101 (2007) 104305.

11) Y. Saito, M. Motohashi, and N. Hayazawa: Appl. Phys. Lett. 88 (2006) 143109.

12) C. Rosenbrad, T. Graf, A. Dommann, and H. V. Kanel: Mater. Res. Soc. Symp. Proc. 533 (1998) 301.

13) P.-H. Wu and D.-S. Lin: Phys. Rev. B 57 (1998) 12421.

14) J. C. Tsang, P. M. Mooney, F. Dacol, and J. O. Chu: J. Appl. Phys. 75 (1994) 8098.

15) M. I. Alonso and K. Winer: Phys. Rev. B 39 (1989) 10056.

16) L. H. Wong, C. C. Wong, J. P. Liu, D. K. Sohn, L. Chan, L. C. Hsia, H. Zang, Z. H. Ni, and Z. X. Shen: Jpn. J. Appl. Phys. 44 (2005) 7922.

17) K. Kutsukake, N. Usami, T. Ujihara, K. Fujiwara, G. Sazaki, and K. Nakajima: Appl. Phys. Lett. 85 (2004) 1335. 